\title{
A New Method to Characterize Changes in the Seasonal Cycle of Snowpack
}

\author{
Amato T. Evan \\ Scripps Institution of Oceanography, University of California, San Diego, La Jolla, California
}

(Manuscript received 30 May 2018, in final form 23 October 2018)

\begin{abstract}
In the western United States, water stored as mountain snowpack is a large percentage of the total water needed to meet the region's demands, and it is likely that, as the planet continues to warm, mountain snowpack will decline. However, detecting such trends in the observational record is challenging because snowpack is highly variable in both space and time. Here, a method for characterizing mountain snowpack is developed that is based on fitting observed annual cycles of snow water equivalent (SWE) to a gammadistribution probability density function. A new method for spatially interpolating the distribution's fitting parameters to create a gridded climatology of SWE is also presented. Analysis of these data shows robust trends in the shape of the annual cycle of snowpack in the western United States. Over the 1982-2017 water years, the annual cycle of snowpack is becoming narrower and more Gaussian. A narrowing of the annual cycle corresponds to a shrinking of the length of the winter season, primarily because snowpack melting is commencing earlier in the water year. Because the annual cycle of snowpack at high elevations tends to be more skewed than at lower elevations, a more Gaussian shape suggests that snowpack is becoming more characteristic of that at lower elevations. Although no robust downward trends in annual-mean SWE are found, robust trends in the shape of the SWE annual cycle have implications for regional water resources.
\end{abstract}

\section{Introduction}

In the western United States, the majority of precipitation falls during the wintertime (Mock 1996), and consequently water supply is heavily dependent upon the amount of precipitation that is stored in mountain snowpack, specifically because snowmelt provides a steady and gradual source of water runoff (Doesken and Judson 1996). As such, year-to-year changes in snow accumulation and melt can have major impacts on water management since the timing of the availability of water is crucial. Thus, the influence of climate change on mountain snowpack is of great scientific and societal interest.

Snowpack is heavily influenced by both precipitation and temperature, and climate change may be reducing mountain snowpack via reductions in wintertime frozen (and potentially liquid) precipitation and early melting (Mote et al. 2005; Barnett et al. 2008; Brown and Robinson 2011; Cayan et al. 2016; Mote et al. 2018), and such changes are expected to continue over the twenty-first century (Diffenbaugh et al. 2013; Krasting et al. 2013; Pierce and Cayan 2013; Mankin and

\footnotetext{
Corresponding author: Amato Evan, aevan@ucsd.edu
}

Diffenbaugh 2015). However, changes in snowpack are not ubiquitous across the western United States. At very high elevations, temperatures are sufficiently cold that planetary warming likely has had little measurable effect on snowpack, or at least on the seasonal maximum snowpack (Cayan 1996). Furthermore, natural climate variability, such as the Pacific decadal oscillation or ENSO, affects snowpack in different regions of the western United States in distinct forms (DeFlorio et al. 2013; Hartmann 2015).

Changes in snowpack are often characterized by measurements snow water equivalent (SWE) on 1 April (e.g., Mote et al. 2018). Although arbitrary, 1 April SWE is a convenient parameter for analysis since there are long-term measurements of SWE made on this date in the western United States, and because many observing sites reach their peak snowpack near this date (Cayan 1996; Bohr and Aguado 2001). However, changes in 1 April SWE are not clearly tied to a single physical process. For example, an anomalously low value of 1 April SWE could result from a lack of winter season precipitation or from anomalously warm temperatures. Furthermore, a time series of SWE on any particular day is inherently noisy and thus is not ideal for detection of secular trends. In this paper, I present an alternative 
method for characterizing snowpack in the western United States that utilizes SWE measurements for the entire water year and that entails fitting gammadistribution probability density functions to the measured annual cycle of SWE. Trend analysis of these parameters elucidates the ways in which the annual cycle of SWE has changed over the historical record.

The remainder of this paper is organized as follows: Data and methods describes the data used (section 2a), a method for fitting gamma-distribution probability density functions to the annual cycle of SWE and evaluating the accuracy of these fits (section 2b), and a method for spatially interpolating the station-level data onto an equal-angle grid (section 2c), along with the corresponding validation statistics (section $2 \mathrm{~d}$ ). Results contain an analysis of the trends in the gamma-distribution fitting parameters for the observational sites (section 3a) and for the spatially interpolated data (section $3 b)$. This is followed by an analysis of the secular changes in the annual cycle of SWE in the discussion section, as well as a comparison with trends from 1 April SWE and other observational metrics of mountain snowpack. The paper ends with a conclusions section that puts the results into a broader context and briefly discusses future work.

\section{Data and methods}

The data and methods section describes the data used, a method to fit gamma distributions to annual time series of SWE, and a method to spatially interpolate the fitted, station-level SWE data onto an equal-angle grid. Validation statistics are also presented.

\section{a. Snow water equivalent data}

Used in this analysis are daily observations of SWE from the Natural Resources Conservation Service Snowpack Telemetry (SNOTEL) network (Serreze et al. 1999). To reduce spurious results, daily data are removed from the record if the absolute value of a single-day change in SWE is greater than $20 \mathrm{~cm}$, a threshold that was chosen through visual analysis of SWE curves from a large number of stations. Furthermore, if any station has more than 30 days of missing data for the period of November-April, the data for that water year are not used. Last, data for a station, for the entire water year, are not used if the measured SWE is zero during every day of January, February, or March. This last criterion stems from the fact that some stations appear to report SWE values of zero when the measurements should be missing. I also use SWE data from one station managed by the California Department of Water Resources (CDWR; Quaking Aspen at $36.12^{\circ} \mathrm{N}, 118.54^{\circ} \mathrm{W}$ ), because this is the only station in the state's network with continuous data

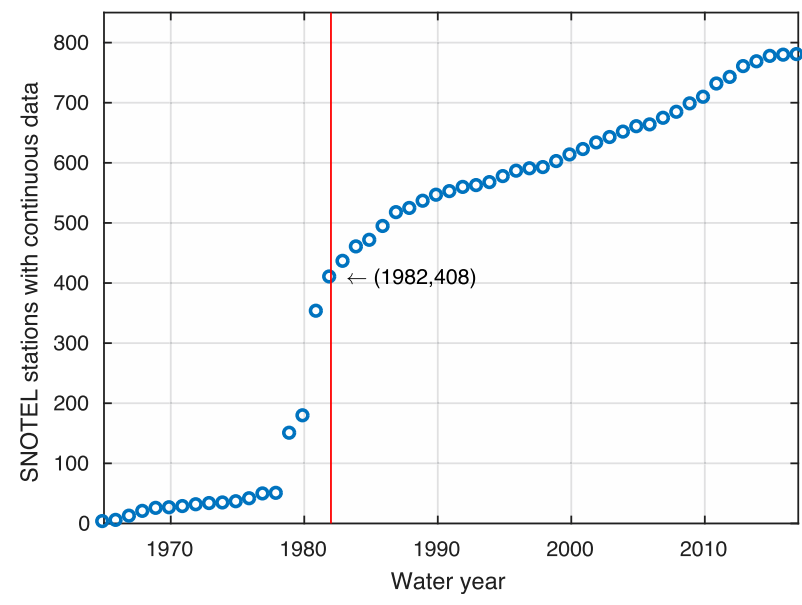

FIG. 1. SNOTEL stations with continuous SWE measurements: the number of SNOTEL sites having continuous data through the 2017 water year (ordinate) as a function of the first year of continuous data (abscissa). The vertical red line demarks the 1982 water year and the corresponding number of sites used in this study (432).

over the time period of interest (after quality control criteria were applied). These data are included in this analysis because this is the only station representing the southern Sierra Nevada. For convenience, throughout this paper I refer to "SNOTEL station" data rather than "SNOTEL and CDWR station" data.

Figure 1 is a plot of the number of SNOTEL sites having continuous measurements as a function of water year, through the 2017 water year. These are sites for which the quality control criteria described above have been applied. There is a large increase in the number of stations with continuous data during 1978-82. Consequently, to balance the need for long-term SWE measurements with the need to have a large number of stations in the analysis, only stations with continuous data from the 1982-2017 water years are used for the trend analysis. This equates to 408 stations having 36 years of continuous SWE measurements. The spatial distribution of the resultant stations is shown in Fig. 2, which includes the location of the one CDWR site. Changing this analysis to include fewer years and more stations did not clearly improve the spatial distribution and representation of the SNOTEL sites to be used for the trend analysis. SWE data from the stations not used in the trend analysis are used for validation of the spatial interpolation method that is described below.

\section{b. SWE curve fitting}

The annual cycle of SWE exhibits characteristics similar to that of the probability distribution function of a gamma distribution that is reflected about the ordinate axis (Fig. 3). The gamma distribution is a continuous 


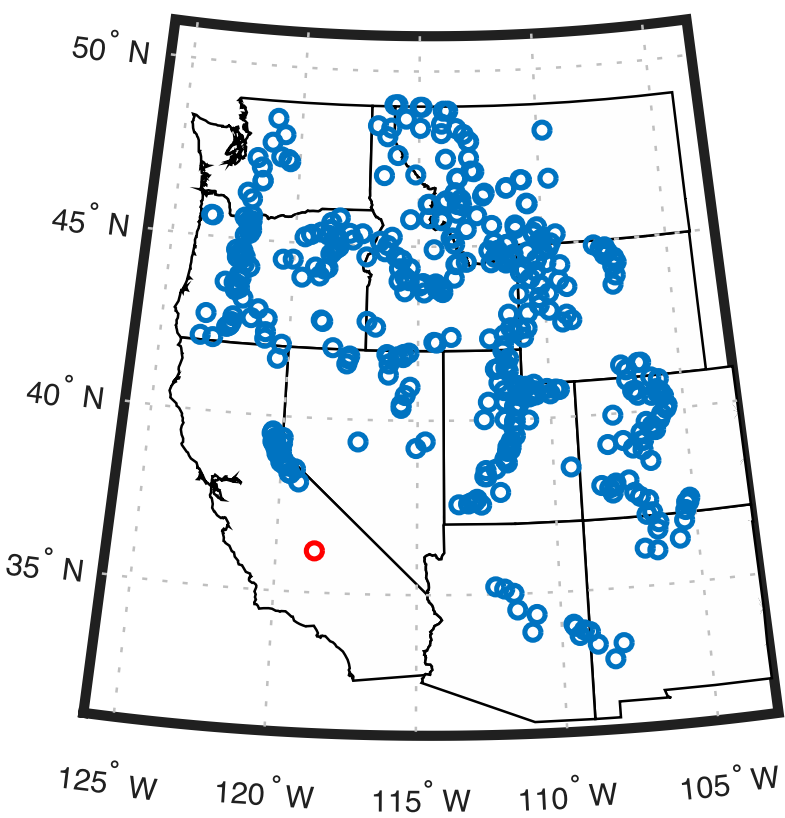

FIG. 2. Spatial distribution of the SNOTEL sites used in this study. These SNOTEL stations have continuous data from the 1982-2017 water years. The CDWR site used in this study is indicated by the red circle.

distribution that is bounded on the left by zero and is positively skewed. The PDF of the gamma distribution is given by

$$
f(x)=\frac{(x / \beta)^{\alpha-1} \exp (-x / \beta)}{\beta \Gamma(\alpha)} .
$$

The shape of the distribution is dependent upon the so-called shape parameter $\alpha$. For $\alpha>1$, the PDF begins at the origin $[f(0)=0]$. At small $\alpha$, the distribution is more skewed to the left, and at larger $\alpha$ the distribution function shifts to the right, eventually approaching a Gaussian. Changes in the size parameter $\beta$ squeeze the distribution to the left (small $\beta$ ) or stretch the distribution to the right (large $\beta$ ). As the distribution is squeezed to the left, the peak of the distribution increases; stretching the distribution to the right reduces the peak height. The gamma distribution has been utilized in hydrological models to estimate the spatial distribution of SWE at the catchment level (see Skaugen and Weltzien 2016 and references therein), which is distinct from the usage presented here. A discussion of the broader use of the gamma distribution in meteorology can be found in Wilks (2011).

To fit Eq. (1) to the annual cycle of SWE, it is necessary to shift the distribution by subtracting an offset $\zeta$ from $x$. Physically, $\zeta$ represents the water yearday in the

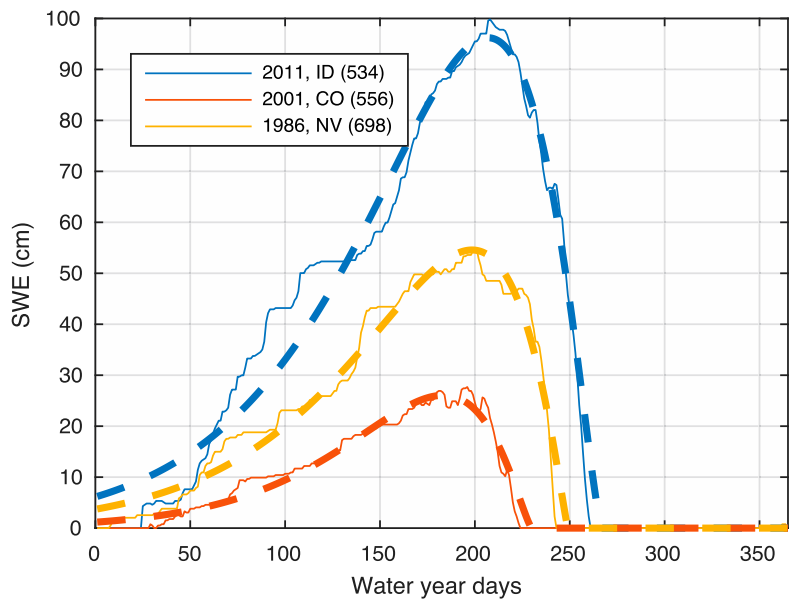

FIG. 3. Examples of the gamma distribution fitted to annual time series of SWE: three time series of SWE for the sites and water years indicated in the legend (thin lines). The state containing each SNOTEL site is also indicated in the legend. Also plotted are the fitted gamma distributions for each site (thick dashed line). The ordinate axis represents SWE $(\mathrm{cm})$, and the abscissa is days after 1 Oct for each respective water year.

spring on which SWE goes to zero. The term $x-\zeta$ must also be multiplied by -1 to reflect the function about the $y$ axis so that SWE gradually increases during the beginning of the water year and then quickly declines in the spring. In addition, the entire distribution function is multiplied by a scaling factor $C$. The modified version of Eq. (1), which will be fit to the annual cycles of SWE, is

$$
f(x)=C \frac{[(\zeta-x) / \beta]^{\alpha-1} \exp [(x-\zeta) / \beta]}{\beta \Gamma(\alpha)},
$$

where $f(x)$ is only valid for $(\zeta-x) \geq 0$.

For each station and water year, $\zeta$ is defined as the first day after the peak in SWE for which SWE drops to zero. The remaining terms in Eq. (2) are then fitted to the daily time series of SWE, again by water year and by station, via the nonlinear least squares trust-region method (Coleman and Li 1996), with lower bounds of 1 for all of the coefficients and upper bounds of 15 and 150 for $\alpha$ and $\beta$, respectively, with no upper bound for $C$. The upper bounds were chosen based on visual inspection of the fits, and these bounds are utilized in less than $0.03 \%$ of the fits for $\alpha$ and less than $0.01 \%$ of the fits for $\beta$.

Figure 3 shows plots of daily mean SWE measurements (thick continuous lines) and the fitted curves (thick dashed line) for three randomly chosen SNOTEL sites and years. For each of these sites the fitted gamma distributions overestimate SWE during the first one to two months of the water year; the tail of the gamma 
distribution falls off more slowly than does the actual SWE (for time in the reverse direction). Otherwise, the gamma distributions provide a good fit to the SWE time series; the correlation coefficient squared $\left(r^{2}\right)$ values for all stations (Fig. 2), averaged by year, are greater than 0.96, and the root-mean-square errors (RMSE) are approximately $6 \%$ of the peak SWE value, where the RMSE is only calculated over the time periods during which the measured SWE is greater than zero.

Figure $4 \mathrm{a}$ is the annual cycle of SNOTEL SWE observations, averaged over all stations and years (blue), and the corresponding mean annual cycle of the fitted SNOTEL SWE data (red). As noted for the individual stations (Fig. 3), the fitted curves overestimate SWE at the beginning of the water year. The fitted SWE curves also slightly underestimate the actual SWE around water yearday 100, and overestimate the magnitude of the peak SWE. The change in springtime SWE is very accurately represented by the gamma distribution. A PDF of the differences between the daily actual and fitted SWE data show a small bias of $-0.29 \mathrm{~cm}$, and a standard deviation of $4.8 \mathrm{~cm}$ (Fig. 4b), which is approximately $10 \%$ of the maximum value of the long-term mean SWE (Fig. 4a). Time series of annual-mean SWE from SNOTEL observations and the fitted SNOTEL data are practically identical (Fig. 4c).

This analysis was repeated by fitting a Weibull and then a lognormal probability distribution function to the SWE data. The mean and standard deviation of the PDF of daily SWE errors (e.g., Fig. 4b) for the lognormal distribution were -1.1 and 6.8 , respectively (not shown), and the mean and standard deviation for the Weibull distribution were -0.77 and 8.8 , respectively (not shown). Thus, among these three skewed distributions, the gamma distribution provided a better fit to the SWE data.

\section{c. Spatial interpolation methods}

The historical SNOTEL sites were identified as being areas where the snowpack measurements correlated well with April-July runoff volumes, and therefore the sites are not necessarily representative of the regional snowpack. Thus, in order to evaluate regional-scale changes in seasonal SWE characteristics, it is useful to spatially interpolate station data to an equal-area grid. Here, spatial interpolation of the data is performed using the hypsometric elevation regression method (Fassnacht et al. 2003). The basis for this method is that SWE is dependent upon temperature and precipitation, and temperature and precipitation are in turn proportional to elevation (Dingman 1981). As such, over a limited geographic region, SWE observations can be regressed onto station elevations in order to determine
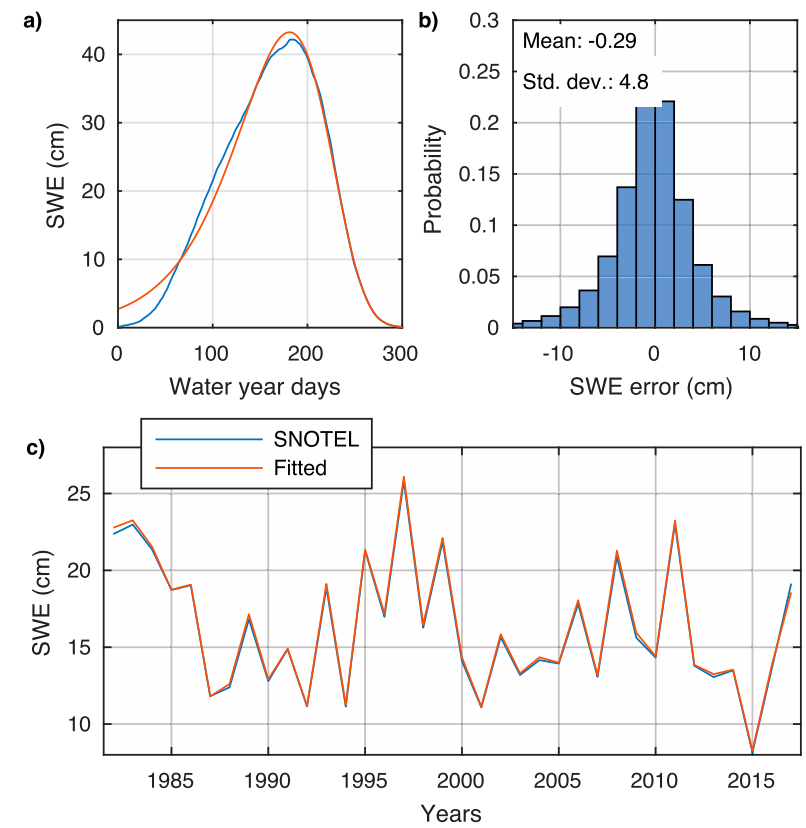

FIG. 4. Comparison of measured and fitted SWE: (a) The longterm mean annual cycle in SWE from SNOTEL and the fitted SNOTEL data. (b) The difference (SNOTEL minus fitted SNOTEL) in daily SWE, for only days for which SNOTEL SWE is greater than zero. The distribution mean and standard deviation are also given. (c) The annual-mean time series of SWE from SNOTEL and the fitted SNOTEL data.

the local SWE lapse rate, and this lapse rate can then be use to estimate the SWE at any elevation in the domain of interest. Similarly, long-term station-mean values of $\alpha, \beta$, and $\zeta$ are statistically significantly correlated with station elevation (Figs. 5a,b,d). Although station-mean $C$ is not correlated with elevation (Fig. 5c) when considering smaller spatial scales $(100 \mathrm{~km})$, there is a positive and statistically significant correlation between $C$ and elevation (not shown).

The first step is to estimate the size of the domain over which the spatial interpolation can be applied. To do so, the decorrelation distance of the parameters used to fit the gamma distributions to the SWE data must be determined. This is the average distance at which the stations' correlation coefficients of the annual time series for each parameter fall to $\exp (-1)$, shown in Fig. 6 . Here, the correlation coefficients for each parameter are plotted as a function of distance between stations (box and whiskers; black line), and the $e$-folding distance is indicated by the red circles. The shape parameter $\alpha$ is the most heterogeneous of the four terms used to fit the gamma distributions, having a decorrelation distance of $45 \mathrm{~km}$ (Fig. 6a), whereas the size parameter $\beta(345 \mathrm{~km})$, scale factor $C(835 \mathrm{~km})$, offset $\zeta(995 \mathrm{~km})$ all have much larger decorrelation distances (Figs. 6b-d). On the basis of the distances in Fig. 6, the hypsometric elevation 
a)
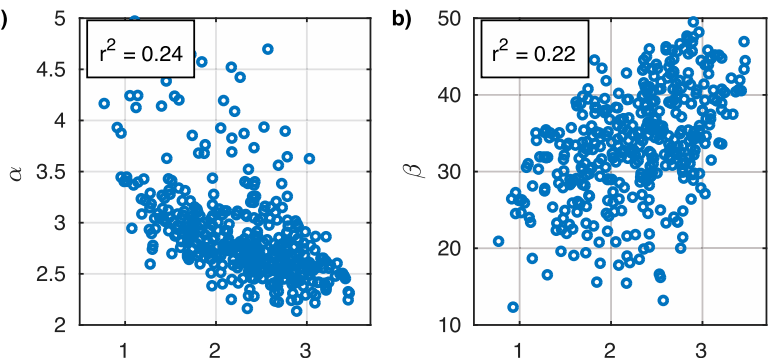

c)

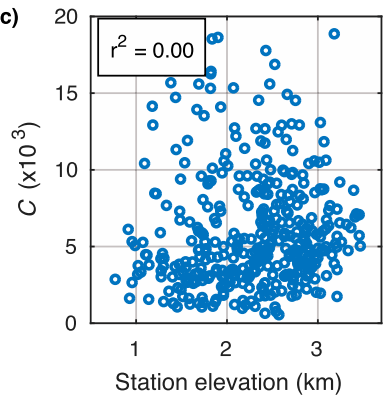

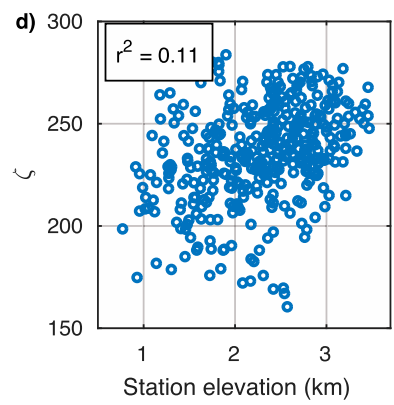

Station elevation $(\mathrm{km})$

FIG. 5. Relationship between station elevation and gammadistribution fitting parameters, as shown by scatterplots of the longterm mean station (a) $\alpha$, (b) $\beta$, (c) $C$, and (d) $\zeta$ as a function of station elevation. Also indicated are the $r^{2}$ values from the correlation coefficients, which, except for $C$, are all statistically significant (significance level $p<0.05$ ).

regressions are applied over a distance of $100 \mathrm{~km}$, which is a compromise between the short decorrelation distance of $\alpha$ and the longer distances of the other parameters.

Spatial interpolation of the fitted SNOTEL data is performed on a $0.25^{\circ} \times 0.25^{\circ}$ equal-angle grid. At each grid point, interpolation of the fitting parameters $(\alpha, \beta$, $C$, and $\zeta$ ) is performed if there are at least five SNOTEL stations within a horizontal distance of $100 \mathrm{~km}$ from the center of the $0.25^{\circ} \times 0.25^{\circ}$ cell. Within this subset of the data, outliers are defined as any parameter with a value outside 2 standard deviations of the sample mean and are not included in the interpolation. Linear least squares regression is used to find the rate of the parameter changes with height, where the parameter is the dependent variable and the station height is the independent variable. However, since the distributions of these parameters are bounded to the left by zero, the elevation regression is conducted on the log of the parameter values. The regression is weighted by the inverse of the distance between each station and the center of the grid cell.

Note that a major difference between this method and Fassnacht et al. (2003) is that here the fitting parameters are being interpolated rather than the actual SWE values. Such an approach leads to fewer errors as the
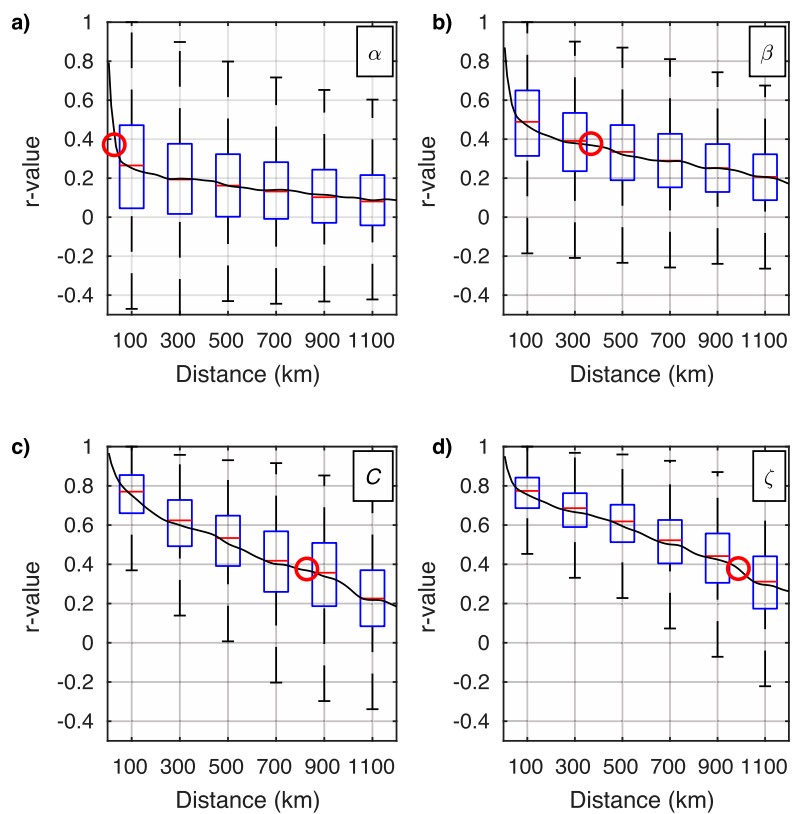

FIG. 6. Decorrelation spatial scales for the four parameters used to fit the gamma distributions: the correlation coefficients $r$ (ordinate axes) as a function of distance (abscissa) for all of the SNOTEL sites. Correlation coefficients are shown as box-andwhisker plots as well as smoothed averaged continuous values (black curves). The red circle indicates the decorrelation distance, which is defined as the distance at which the correlation coefficient falls to $\exp (-1)$.

interpolation is performed by season on the fitting parameters, rather than by day on the measured SWE; daily values of SWE can contain missing or spurious values, even after quality controls are applied. Furthermore, I note that the methods of Fassnacht et al. (2003) included an additional step of adding the inverse distance weighted mean of the regression residuals to the interpolated SWE values. I found that this step did not improve the accuracy of the interpolated data and thus did not include it here (not shown). Furthermore, since the single CDWR station is not within $100 \mathrm{~km}$ of at least four SNOTEL sites, there was not any spatially interpolated data in Southern California.

\section{d. Spatial interpolation validation}

The spatial interpolation technique is validated using data from 396 SNOTEL sites that are not included in the analysis because their data record was not continuous from 1982 to 2017 (Fig. 1). The spatial distribution of these validation SNOTEL sites was similar to that shown for the sites used to perform the spatial interpolation (Fig. 2). Here, the gamma-distribution fitting parameters from Eq. (2) of $\alpha, \beta, C$, and $\zeta$ are spatially interpolated to the validation SNOTEL sites in a manner identical to that described in section $2 \mathrm{c}$. 

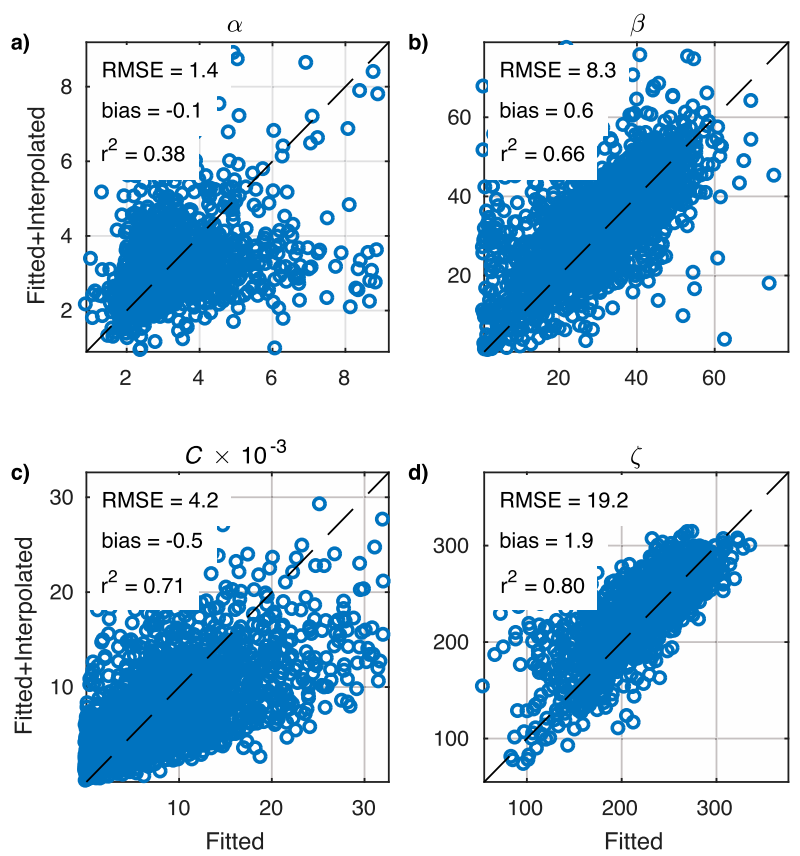

FIG. 7. Scatterplots of the gamma-distribution parameters from the SNOTEL validation stations and from the spatially interpolated data. Plotted are the station-mean $\alpha, \beta, C$, and $\zeta$ from SNOTEL sites that were not used to create the gridded SWE data (abscissa) and the estimates for those same values from the gridded data, interpolated to the horizontal location and elevation of the SNOTEL sites (ordinate). Also given are the RMSE, bias, and $r^{2}$ values for the data in the plots.

These interpolated parameters are then used to reconstruct an SWE time series at each station.

In Fig. 7 are scatterplots of the fitting parameters for the validation SNOTEL sites ("fitted"; abscissa), and the spatially interpolated data ("fitted+interpolated"; ordinate). Also indicated in each are the RMSE, bias, and $r^{2}$ value for the two data. Consistent with the decorrelation distances (Fig. 6), $r^{2}$ values for the interpolated $\alpha$ and $\beta$ (Figs. 7a,b) are smaller than those for $C$ and $\zeta$ (Figs. 7c,d). The RMSEs for $\alpha, \beta$, and $C$ are all on the order of $10 \%$ of their respective mean values, whereas the RMSE for $\zeta$ is on the order of $1 \%$ of the mean. The biases for all parameters are on the order of $1 \%$ of their respective means. Year-to-year changes in the interpolated parameters are highly correlated with the parameters fitted directly from the SNOTEL SWE data where the $r^{2}$ values for the station-averaged time series are 0.77 for $\alpha, 0.95$ for $\beta$, and 0.97 for both $C$ and $\zeta$ (Fig. 8).

The long-term mean annual cycle of SWE from the fitted and interpolated data is very similar to that from the actual SNOTEL observations (Fig. 9a), which is a consequence of the relatively low biases in the fitting parameters (Fig. 7). However, a histogram of the differences in daily SWE from SNOTEL observations and a)
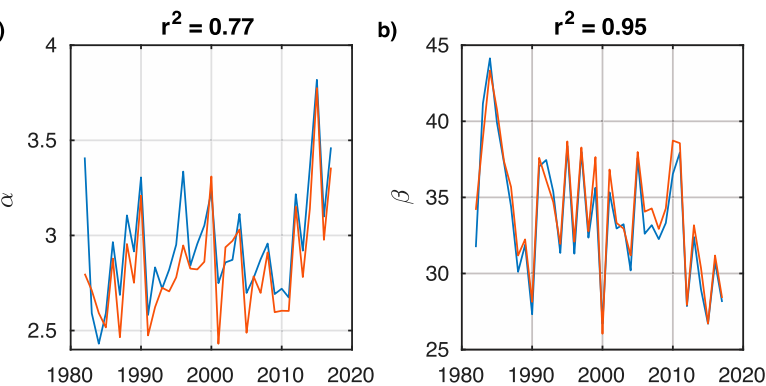

c)
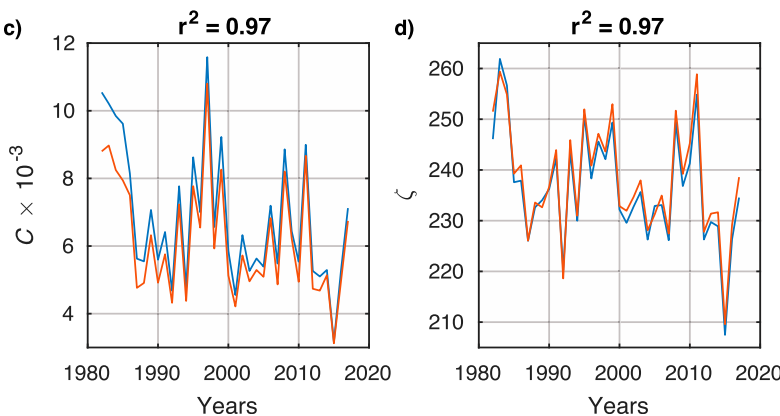

FIG. 8. Time series of gamma fitting coefficients (blue) with those from interpolation (red), for the SNOTEL validation sites. The time series in each plot are of parameters averaged over all SNOTEL stations that are used for validation of the interpolation method. The $r^{2}$ values for the time series are also given. Note that for these plots the year-to-year changes in each parameter are related to changes in the numbers of stations with available data.

the fitted and interpolated data shows a wide distribution of errors in SWE (Fig. 9b), where the 1- $\sigma$ error is approximately $21 \mathrm{~cm}$ (the histogram in Fig. 9b was constructed using only days for which the measured SWE was nonzero). The RMSE is dominated by errors associated with the spatial interpolation technique; RMSE errors from the gamma-distribution fitting step are a factor of 4 smaller $(4.8 \mathrm{~cm}$; Fig. $4 \mathrm{~b})$. The time series of annual-mean SWE from the validation-site SNOTEL data and that from the fitted and interpolated data show a high level of agreement (Fig. 9c; $r^{2}=0.97$ ). Maps of the biases and RMSEs, normalized by each station's mean SWE, do not exhibit spatial structure of the errors in the fitted and interpolated SWE data (not shown).

\section{Results}

In the previous section, I described and validated the method for fitting gamma distributions to annual time series of SWE observations from SNOTEL, as well as the method for spatially interpolating those fitting parameters onto a $0.25^{\circ} \times 0.25^{\circ}$ equal-angle grid. In this section, the fitted data and the fitted and interpolated data are in turn used to identify long-term trends in western U.S. snowpack. 

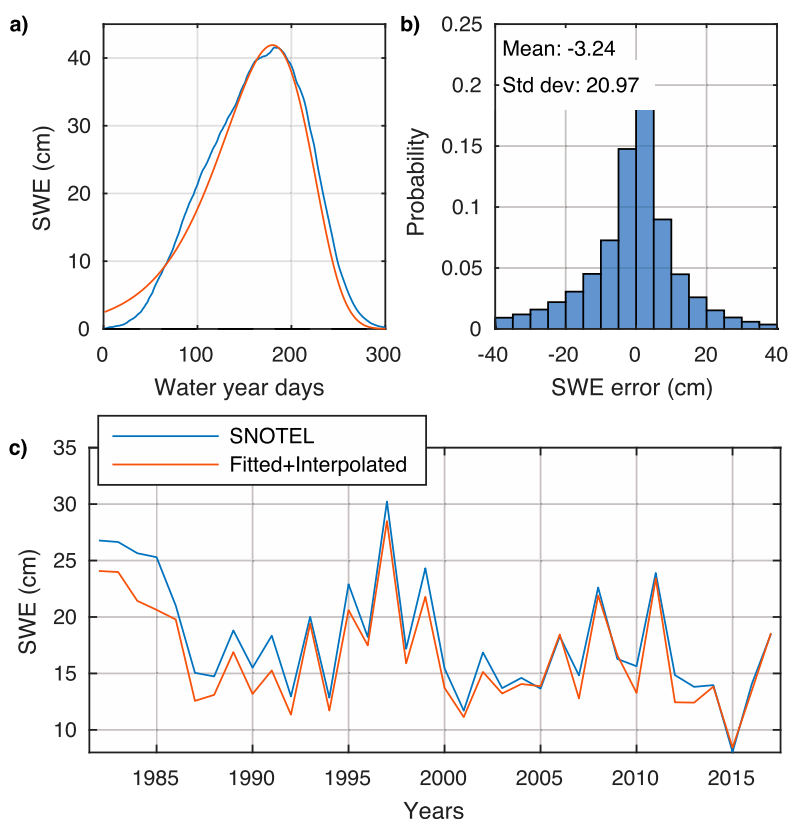

FIG. 9. As in Fig. 4 except that here measured SNOTEL data are compared with the fitted and interpolated SNOTEL data. Only validation SNOTEL sites are used to generate these plots.

\section{a. Trends at SNOTEL sites}

First, trends are calculated for the gamma-distribution fitting parameters $\alpha, \beta, C$, and $\zeta$ [Eq. (1)], but only for the SNOTEL sites with continuous data from 1982 to 2017 (Figs. 1 and 2). Here, the linear 36-yr change in each parameter is calculated for each SNOTEL station by multiplying the linear trend $\left(\mathrm{yr}^{-1}\right)$ by $36 \mathrm{yr}$. Statistical significance of trends is calculated via a Mann-Kendall test. Figure 10 shows histograms of the stations' trends for each parameter. Here, the percentage of stations with positive, negative, and statistically significant 36-yr changes are indicated in the legend for each plot. Figure 11 has maps showing the spatial distribution of those trends among the SNOTEL stations. Figure 12 gives time series of the each parameter, averaged over all stations, as well as a plot of the corresponding linear trend. Also indicated in Fig. 12 are the $95 \%$ confidence intervals on these linear trends.

A positive 36-yr change in $\alpha$ was calculated for a majority of the SNOTEL sites used in the trend analysis (Fig. 10a), with nearly $15 \%$ of those stations showing a statistically significant change. The annual cycle of SWE becomes more Gaussian with increasing $\alpha$, and in general $\alpha$ increases with station elevation (Fig. 5a). Thus, a majority of SNOTEL sites are exhibiting a less skewed annual cycle in SWE, or an annual cycle that is more characteristic of lower-elevation conditions. The spatial distribution of this upward trend in $\alpha$ shows that while
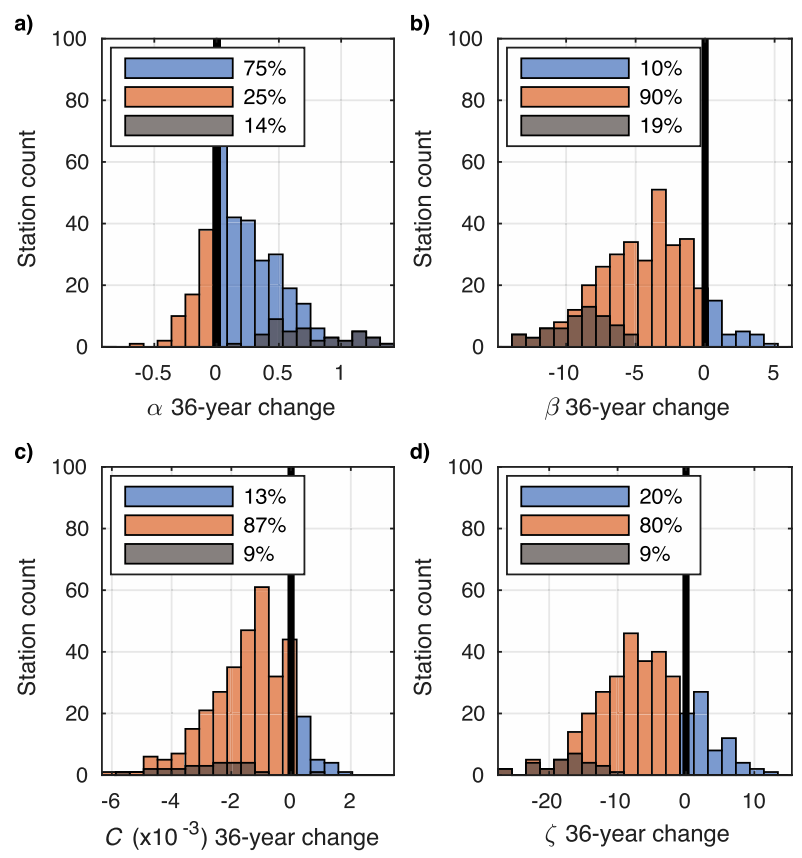

FIG. 10. Histograms of trends in the fitting parameters at the SNOTEL stations, giving linear 36-yr changes in the fitting parameters (a) $\alpha$, (b) $\beta$, (c) $C$, and (d) $\zeta$. The legends give the percentage of positive (blue), negative (orange), and statistically significant (gray) linear changes for each parameter.

the upward trends are spread throughout the western United States, the largest positive trends appear to be most prevalent at SNOTEL sites in Utah, Colorado, and Southern California (Fig. 11a). The trend in the annualmean time series of $\alpha$, averaged over these SNOTEL sites, also exhibits a positive upward trend (Fig. 12a), where the corresponding 36-yr linear change of 0.25 $( \pm 0.24)$ is statistically significant.

Next, $90 \%$ of the SNOTEL sites examined here have a negative change in $\beta$, with $19 \%$ having a statistically significant negative change (Fig. 10b). The annual cycle of SWE is squeezed (in time) to the right with decreasing $\beta$, which can be interpreted as a reduction in the length of time that SWE is greater than zero, or a reduction in the length of the winter season. Consequently, the length of the winter season is decreasing for an overwhelming majority of these SNOTEL sites, and this is the most consistent result among all of the parameters in Fig. 10. The spatial distribution of the trends in $\beta$ shows negative values more or less uniformly spread among the SNOTEL sites, although the trends are closer to zero, and in some cases positive, in the region of southwestern Montana (Fig. 11b). The time series of $\beta$, averaged over all stations, exhibits a statistically significant negative trend, having a 36-yr linear change of $4.3 \pm 4.1$ (Fig. 12b). 


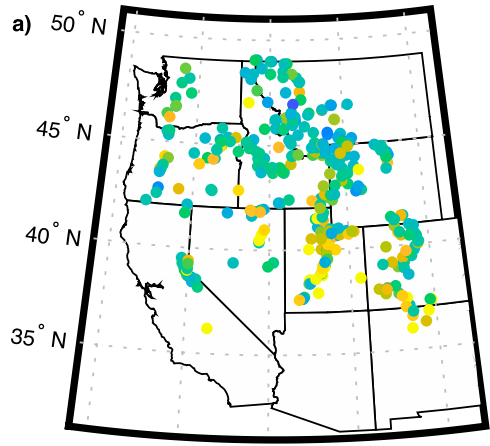

$125^{\circ} \mathrm{W} 120^{\circ} \mathrm{W} 115^{\circ} \mathrm{W} 110^{\circ} \mathrm{W} 105^{\circ} \mathrm{W}$

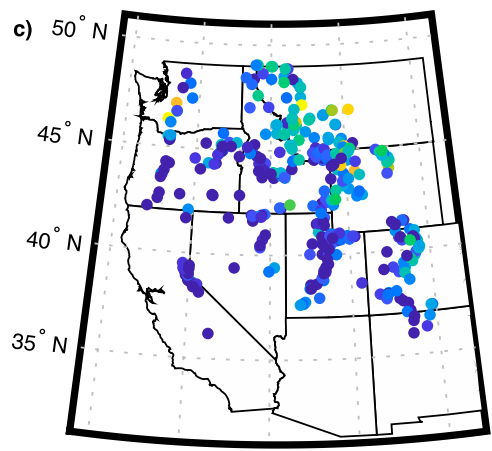

$125^{\circ} \mathrm{W} 120^{\circ} \mathrm{W} 115^{\circ} \mathrm{W} 110^{\circ} \mathrm{W} 105^{\circ} \mathrm{W}$
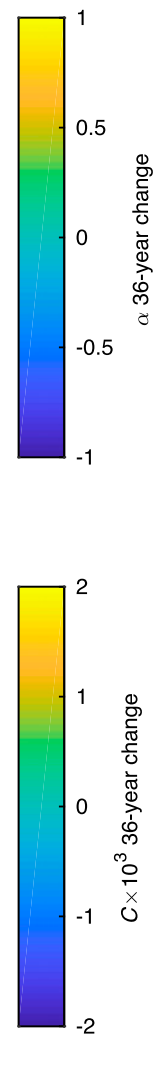

FIG. 11. Maps of the spatial distributions of 36-yr changes in the (a) $\alpha$, (b) $\beta$, (c) $C$, and (d) $\zeta$ parameters used to fit gamma distributions to the SNOTEL data.

A majority of these SNOTEL sites (87\%) also show a negative change in $C$, with $9 \%$ of the stations having a statistically significant negative change (Fig. 10c). The spatial distribution of this change in $C$ is heavily weighted toward stations in the southwest portion of the western United States, with positive trends in $C$ to the northeast (Fig. 11c). Consequently, although the time series of $C$, averaged over the SNOTEL stations, shows a negative trend, this linear change is not statistically significant (Fig. 12c). As $C$ is highly correlated with annual-mean SWE, this result is consistent with the annual-mean time series of SWE in Fig. 4b, where the 36 -yr linear change is $-4.0 \pm 4.8 \mathrm{~cm}$.

Last, a majority of these SNOTEL sites $(80 \%)$ also show a reduction in $\zeta$, although only $9 \%$ of these stations exhibit a statistically significant change (Fig. 10d). By themselves, decreases in $\zeta$ indicate that the SWE distribution is shifting to the left (earlier in the water year), but in conjunction with an increase in $\beta$ (Fig. 10b) they suggest that the winter season is being compressed, at least partially because of an earlier spring snowmelt. The spatial distribution of the trends in $\zeta$ is highly heterogeneous but in general can be characterized as negative
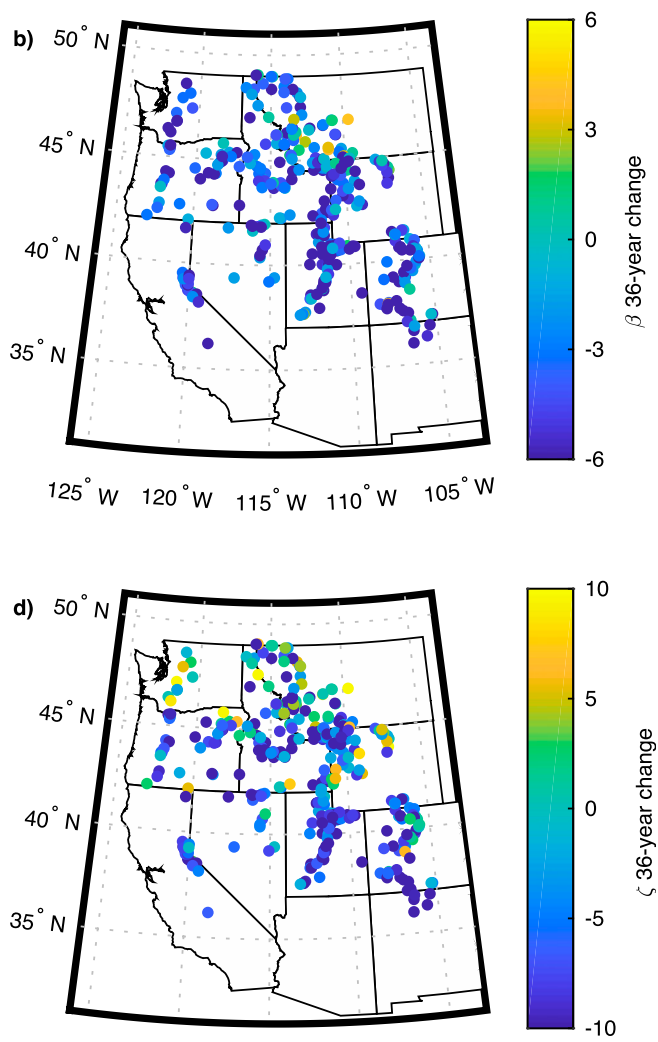

$125^{\circ} \mathrm{W} 120^{\circ} \mathrm{W} 115^{\circ} \mathrm{W} 110^{\circ} \mathrm{W} 105^{\circ} \mathrm{W}$

\section{.}

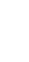


a)

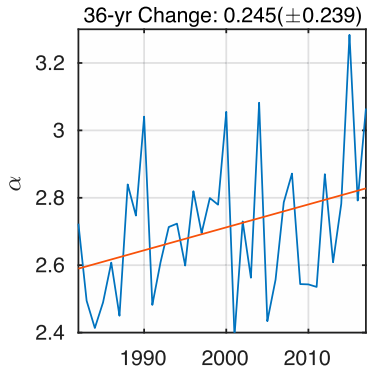

c)

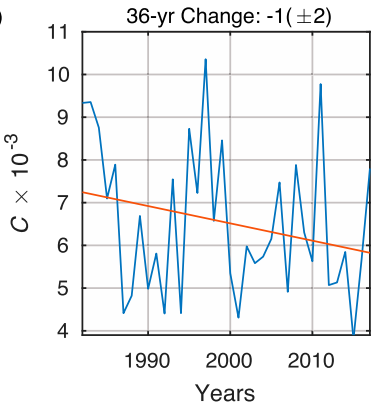

b)

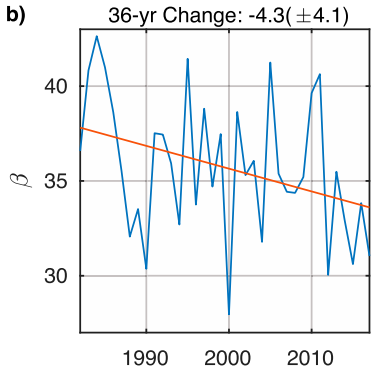

d)

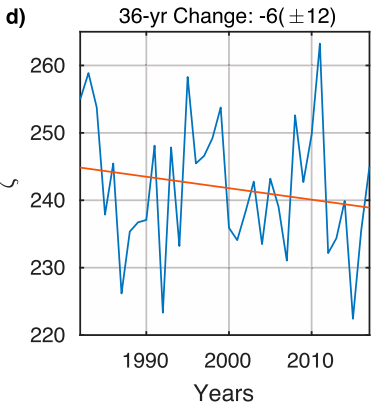

FIG. 12. Time series (blue) and linear trends (red) of the parameters used to fit gamma distributions. All time series and trends are averages over all SNOTEL stations having continuous data from 1982 to 2017 (Figs. 1, 2). Also shown are the magnitudes and $95 \%$ confidence intervals (in parentheses) of the 36-yr change in each parameter as based on the linear trend.

With regard to $\alpha, 83 \%$ of the spatially interpolated data exhibit an upward trend in this parameter, with $18 \%$ having a statistically significant trend (Fig. 13a). These positive trends in $\alpha$ are mainly contained to regions in the southern half of the western United States, with a broad area of trends that are much closer to zero in the northeast (Fig. 14a). The mean time series of $\alpha$ also exhibits a statistically significant upward trend (Fig. 15a), where the 36 -yr change is $-0.6( \pm 0.4)$, consistent with the SNOTEL data (Fig. 10a). However, in the spatially interpolated data, the trend magnitude is twice as large as that for the fitted-only data, a result of the larger positive values for the 2014 and 2017 water years in the spatially interpolated data.

For $\beta, 92 \%$ of the interpolated data have downward trends, and $24 \%$ have trends that are statistically significant (Fig. 13b), which is just slightly higher than that for the fitted SNOTEL data (Fig. 10b). Also, similar to the SNOTEL-only time series (Fig. 12b), the time series of $\beta$ for the interpolated data has a statistically significant downward trend (not shown), and the 36-yr change is $-5.4( \pm 3.8)$. These downward trends in $\beta$ are widespread throughout the domain (Fig. 14b), although there are pockets where the trends are positive, most notably in the Cascade Mountains (i.e., the western edge of Oregon and Washington states, particularly along the border).
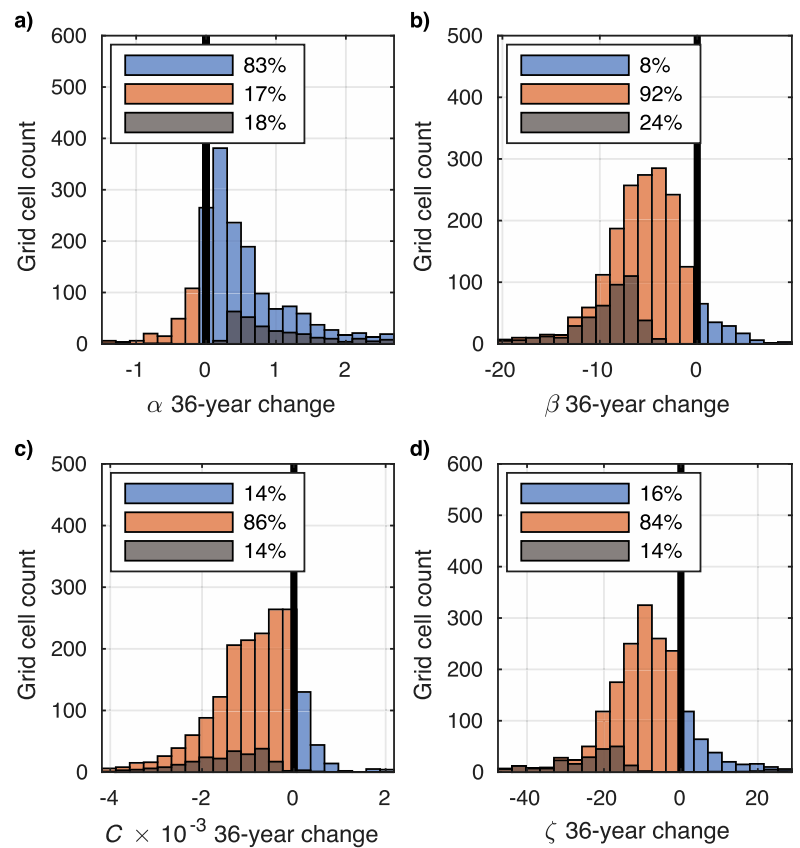

FIG. 13. As in Fig. 10 except that trends are calculated from the fitted and interpolated data.

Also, similar to the SNOTEL-only time series (Fig. 12b), the time series of $\beta$ for the interpolated data has a statistically significant downward trend (Fig. 15b). However, this trend is clearly resultant from anomalously high values for $\beta$ during the 1980s, and low values after 2011 .

A majority $(86 \%)$ of the interpolated data have a negative trend in $C$, with $14 \%$ of the grid cells exhibiting a statistically significant trend (Fig. 13c), which is nearly identical to the case for the SNOTEL trends (Fig. 10c). However, from Fig. 14c these trends are much more uniform throughout the region, with exceptions being most of Montana and again the coastal border region between Oregon and Washington states. This is slightly distinct from the SNOTEL trend map (Fig. 11c), which exhibits a stronger gradient in the trends in the northeast-to-southwest direction. Similar to the case for the SNOTEL data (Fig. 12c), the time series of $C$ exhibits a downward trend that is not statistically significant (Fig. 15c).

Similar to $C$, a majority $(84 \%)$ of the spatially interpolated data also exhibit a downward trend in $\zeta$, with $14 \%$ having a statistically significant trend (Fig. 13d), which is slightly higher than the trends for the fitted SNOTEL data (Fig. 10d). While the downward trends in $\zeta$ are widespread throughout the domain, there are several regions with positive trends in $\zeta$, including eastern Wyoming, eastern Colorado (near $105^{\circ} \mathrm{W}$ and $40^{\circ} \mathrm{N}$ ), and southwestern Oregon (Fig. 14d). Last, the annual-mean time series of $\zeta$ has a downward trend that is not 

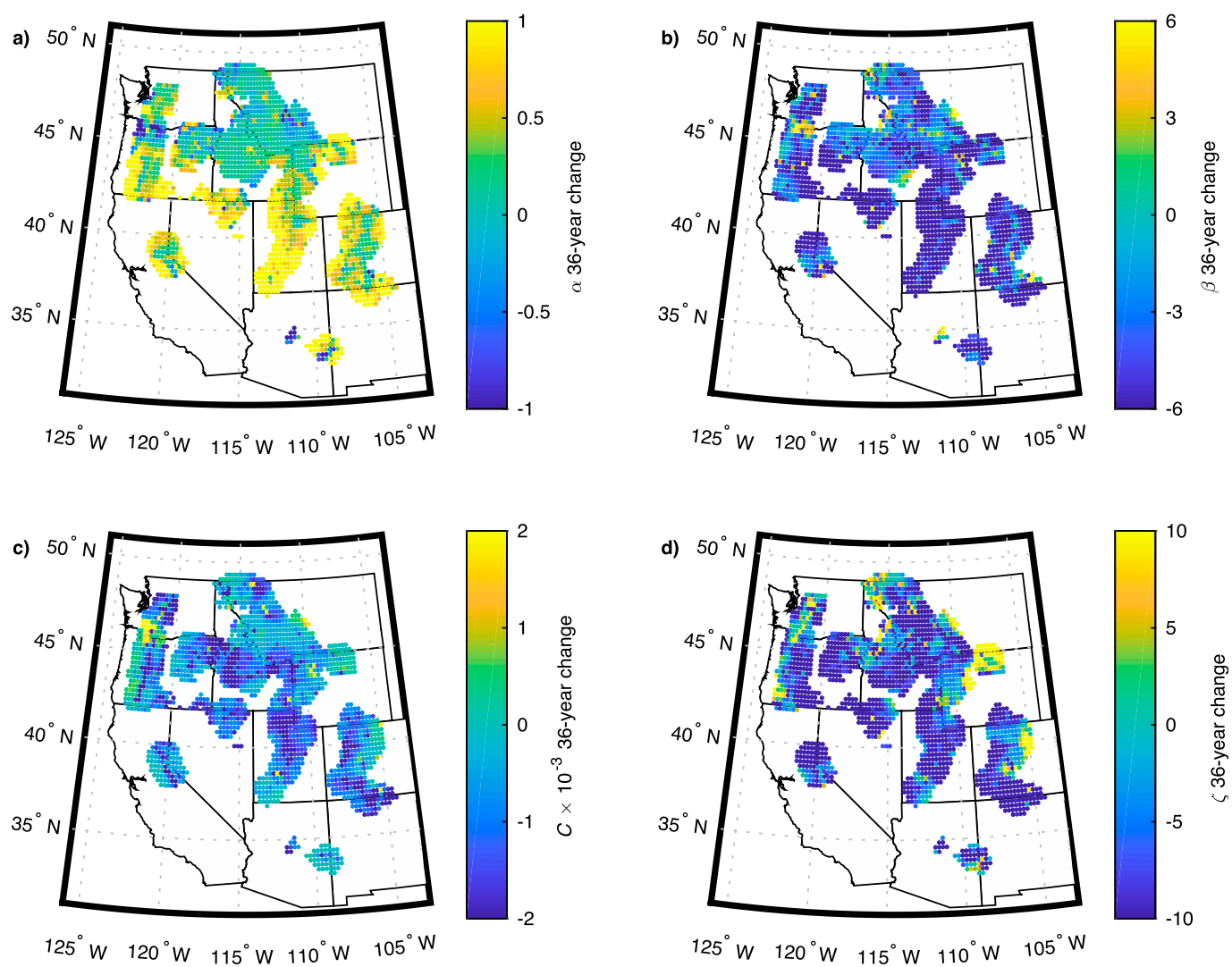

FIG. 14. As in Fig. 11 except that trends are calculated from the fitted and interpolated data.

statistically significant (Fig. 15d), as is the case for the fitted SNOTEL time series (Fig. 12d).

\section{Discussion}

What is the benefit of analyzing the parameters of a distribution used to fit the annual cycle of SWE, rather than direct analysis of the SWE data itself? First, by fitting gamma-distribution probability density functions to the SWE annual cycles one is able to minimize the influence of missing and spurious data on trend analysis. This is a major advantage of the approach since occasional missing data are common in the SNOTEL record.

Next, the trends of the individual parameters can be used to quantify secular changes in the shape of the annual cycle of SWE. For example, plotted in Fig. 16a is the annual cycle of SWE (blue line), calculated via Eq. (2), using the long-term mean and spatially averaged values of the parameters calculated from the fitted SNOTEL data (Fig. 12). Also plotted are SWE annual cycles calculated from the parameter values for the trend lines in Fig. 12, for the 1982 and for 2017 water year (gray-shaded region). The difference between these two annual cycles
(2017 minus 1982; red) represents the 36-yr linear change in the annual cycle of SWE (red line), based on trends in the fitting parameters. The net effect of the increase in $\alpha$, and the reductions in $\beta, C$, and $\zeta$, is a large reduction in SWE after water yearday 150 (27 February), which peaks in magnitude $(-16 \mathrm{~cm})$ late in the season (water yearday 221, or 9 May).

A similar plot of the change in the SWE annual cycle, but constructed using the trends in the fitted and spatially interpolated SNOTEL data (Fig. 15), shows an identical pattern in the linear change in SWE (Fig. 16b). Thus, the trends in the shape of the annual cycle of SWE from the fitted data (Fig. 16a) are unlikely to result from the spatial heterogeneity of the SNOTEL sites. I note that SWE values for the fitted and spatially interpolated SNOTEL data are smaller than those for the fitted data because the interpolated data encompass more lower-elevation terrain. While the long-term trend in annual-mean SWE is not significant (Fig. 12c), this analysis of the seasonal cycle (Fig. 16) shows that there is a general reduction in SWE, but that this reduction is loaded onto the end of the season, and is manifest as a systematically earlier snowmelt. This result is consistent with an analysis of the seasonality of trends in snowpack across the West 

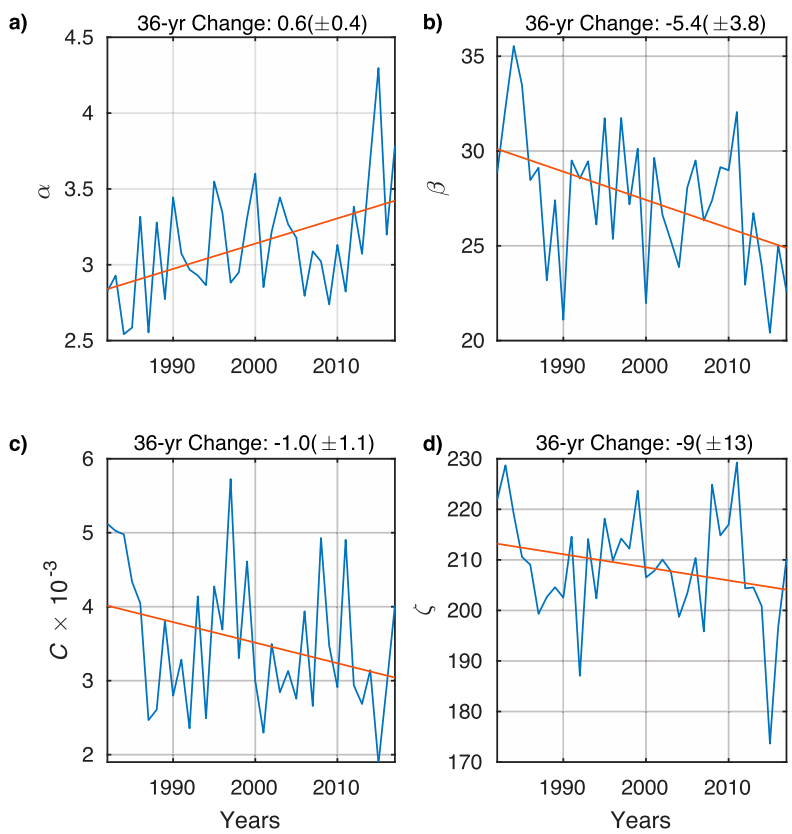

FIG. 15. As in Fig. 12 except that the time series and trends are calculated from the fitted and interpolated data.

based on output from a hydrological model spanning 1915-2014 (Mote et al. 2018), with trends in streamflow measurements (Cayan et al. 2001), and with studies examining output from GCMs (Stewart et al. 2004; Mankin and Diffenbaugh 2015; Gergel et al. 2017). The unique value of the approach developed here is that these results were determined from 36 years of observational data.

In addition, analysis of these fitting parameters provides an opportunity to identify secular trends in the annual cycle of SWE with fewer years of data than would be needed for more typical metrics of snowpack. For example, a time series of 1 April SWE, averaged over SNOTEL station observations (Fig. 2) and over 1982-2017, exhibits a 36-yr trend of $-9.5 \mathrm{~cm}$, which is not statistically significant (Mann-Kendall $p$ value is 0.23 ). Thus, an examination of 1 April SWE from SNOTEL measurements alone would not demonstrate a regional, secular change in snowpack. However, the statistically significant trends in the fitting parameters $\alpha$ and $\beta$ do demonstrate robust regional changes in snowpack over the same time period (Figs. 12a,b). This result is broadly consistent with another study of the trends in various features describing mountain snowpack (Pierce and Cayan 2013). Furthermore, the time series of 1 April SWE (not shown) is highly correlated with that of $C$ (Fig. 4c; $r$ value is 0.95 ). Thus, it is unlikely that there would be a significant trend in 1 April SWE, because there was not a statistically significant trend in $C$.
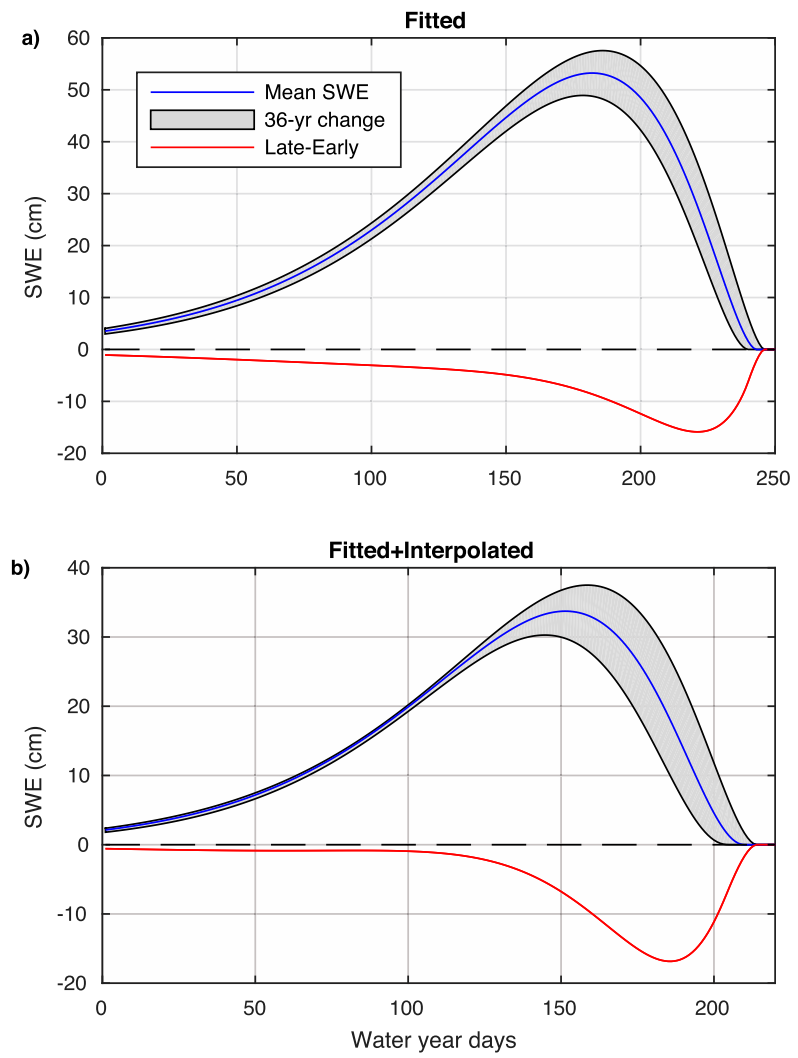

FIG. 16. Time series of the 36-yr change in the annual cycle of SWE as based on (a) the mean of the SNOTEL sites (Fig. 12) and (b) the fitted and interpolated data (not shown): the mean SWE (blue), the maximum and minimum SWE over a 36-yr time period as based on the linear trends (gray-shaded region), and the difference between the maximum and minimum SWE (red), which can also be interpreted as the 36-yr linear trend in SWE.

Trend analysis of other directly measured quantities gives similar results. For example, a time series of the number of days during which SWE $>0$, calculated for each SNOTEL station over 1982-2017 and then averaged over all sites, can be interpreted as a measure of the length of the winter season. This time series (not shown) exhibits a $36-y r$ linear trend of -6.0 days, which is also not statistically significant (Mann-Kendall $p$ value is 0.26). A SNOTEL time series of the water yearday when SWE is at its maximum value, again calculated for each site for 1982-2017 and then averaged over all stations, can be interpreted as a time series of when seasonal melting commences. The 36-yr linear change in this date is -10.6 days (not shown) and is also not statistically significant (Mann-Kendall $p$ value is 0.09).

The purpose of these comparisons is not to suggest that a trend analysis of the fitting parameters is necessarily better than a trend analysis of directly measured quantities like 1 April SWE. Rather, it suggests that a trend analysis of the fitting parameters in Eq. (2) 
provides a method for quantifying how the shape of the seasonal cycle of SWE changes over time and that such an analysis may lead to earlier detection of secular trends in SWE.

\section{Conclusions}

In this paper, methods to fit the annual cycle of observed SWE to a gamma-distribution probability density function [Eq. (2)] and a method to spatially interpolate these data at the regional scale are described. Then, a trend analysis is conducted on the parameters used to fit these probability density functions. From this trend analysis, there is robust evidence that over the western United States the annual cycle of SWE is growing less skewed and is becoming narrower. This is a consistent result seen in the data from the SNOTEL stations (Figs. 10-12) and in the spatially interpolated data (Figs. 13-15). Furthermore, the signs of the trends in these four parameters all are characteristic of declining snowpack. For example, lower-elevation SNOTEL sites tend to have a more Gaussian annual cycle of SWE, whereas higher elevation sites are more strongly skewed (Fig. 5a). Thus, these trends suggest that over time the annual cycle of SWE is becoming more characteristic of lower elevations.

The positive trends in $\beta$ and negative trends in $\zeta$ suggest that the annual cycle is becoming narrower or the winter season is shrinking in duration and that spring snowmelt is happening earlier in the season. The progressively earlier spring snowmelt will have ramifications for regional water management (Hamlet and Lettenmaier 1999; Barnett et al. 2005), as well as regional wildfire activity (Westerling et al. 2006). This result is also consistent with climate model simulations of increasing $\mathrm{CO}_{2}$ (Lettenmaier and Gan 1990; Stewart et al. 2004); thus, these results could be interpreted as direct observational evidence that planetary warming is currently affecting water resources in the West.

The methodological approach described here is useful because trends in the parameters $\alpha, \beta$, and $\zeta$ describe how the annual cycle of SWE is changing over time. It is possible that analysis of these parameters allows for the detection of secular changes in observed snowpack over shorter periods when compared with other metrics like 1 April SWE. Furthermore, an analysis of the seasonal cycle of SWE in climate models may be useful in terms of model evaluation and to better understand historical and future forced changes in snowpack. More rigorous testing of this hypothesis is needed. Last, future work will examine how the parameters $\alpha, \beta$, and $\zeta$ are affected by environmental features such as precipitation and temperature as well as other meteorological processes.
Acknowledgments. The author acknowledges Joel Gratz for advice on interpreting SNOTEL data and three anonymous reviewers for comments on an earlier typescript version of this paper. Funding for this work was provided by NOAA/CPO Grant NA17OAR4310163 and California Electric Program Investment Charge Grant EPC-15-036 to the University of California. These data and related items of information have not been formally disseminated by NOAA and do not represent any agency determination, view, or policy. Data are provided by the California Department of Water Resources (http://cdec. water.ca.gov/snow/current/snow/index.html) and the National Resources Conversation Service (http://www. wcc.nrcs.usda.gov/snow/). The data used for this trend analysis are archived and are accessible via the Pangea Open Access library (https://doi.pangaea.de/10.1594/ PANGAEA.896396).

\section{REFERENCES}

Barnett, T. P., J. C. Adam, and D. P. Lettenmaier, 2005: Potential impacts of a warming climate on water availability in snowdominated regions. Nature, 438, 303, https://doi.org/10.1038/ nature04141.

- , and Coauthors, 2008: Human-induced changes in the hydrology of the western United States. Science, 319, 1080-1083, https://doi.org/10.1126/science.1152538.

Bohr, G. S., and E. Aguado, 2001: Use of April 1 SWE measurements as estimates of peak seasonal snowpack and total coldseason precipitation. Water Resour. Res., 37, 51-60, https:// doi.org/10.1029/2000WR900256.

Brown, R., and D. Robinson, 2011: Northern Hemisphere spring snow cover variability and change over 1922-2010 including an assessment of uncertainty. Cryosphere, 5, 219-229, https:// doi.org/10.5194/tc-5-219-2011.

Cayan, D. R., 1996: Interannual climate variability and snowpack in the western United States. J. Climate, 9, 928-948, https:/ doi.org/10.1175/1520-0442(1996)009<0928:ICVASI>2.0.CO;2.

- S. A. Kammerdiener, M. D. Dettinger, J. M. Caprio, and D. H. Peterson, 2001: Changes in the onset of spring in the western United States. Bull. Amer. Meteor. Soc., 82, 399-416, https:// doi.org/10.1175/1520-0477(2001)082<0399:CITOOS >2.3.CO;2.

—, M. D. Dettinger, D. Pierce, T. Das, N. Knowles, F. M. Ralph, and E. Sumargo, 2016: Natural variability, anthropogenic climate change, and impacts on water availability and flood extremes in the western United States. Water Policy and Planning in a Variable and Changing Climate, K. A. Miller et al., Eds., CRC Press, 17-42.

Coleman, T. F., and Y. Li, 1996: An interior trust region approach for nonlinear minimization subject to bounds. SIAM J. Optim., 6, 418-445, https://doi.org/10.1137/0806023.

DeFlorio, M. J., D. W. Pierce, D. R. Cayan, and A. J. Miller, 2013: Western U.S. extreme precipitation events and their relation to ENSO and PDO in CCSM4. J. Climate, 26, 4231-4243, https://doi.org/10.1175/JCLI-D-12-00257.1.

Diffenbaugh, N. S., M. Scherer, and M. Ashfaq, 2013: Response of snow-dependent hydrologic extremes to continued global warming. Nat. Climate Change, 3, 379, https://doi.org/10.1038/ nclimate1732. 
Dingman, S. L., 1981: Elevation: A major influence on the hydrology of New Hampshire and Vermont, USA. Hydrol. Sci. Bull., 26, 399-413, https://doi.org/10.1080/02626668109490904.

Doesken, N., and A. Judson, 1996: The Snow Booklet: A Guide to the Science, Climatology, and Measurement of Snow in the United States. Colorado State University Publications, 84 pp.

Fassnacht, S., K. Dressler, and R. Bales, 2003: Snow water equivalent interpolation for the Colorado River Basin from Snow Telemetry (SNOTEL) data. Water Resour. Res., 39, 1208, https://doi.org/10.1029/2002WR001512.

Gergel, D. R., B. Nijssen, J. T. Abatzoglou, D. P. Lettenmaier, and M. R. Stumbaugh, 2017: Effects of climate change on snowpack and fire potential in the western USA. Climatic Change, 141, 287-299, https://doi.org/10.1007/s10584-017-1899-y.

Hamlet, A. F., and D. P. Lettenmaier, 1999: Effects of climate change on hydrology and water resources in the Columbia River basin. J. Amer. Water Resour. Assoc., 35, 1597-1623, https://doi.org/10.1111/j.1752-1688.1999.tb04240.x.

Hartmann, D. L., 2015: Pacific sea surface temperature and the winter of 2014. Geophys. Res. Lett., 42, 1894-1902, https:// doi.org/10.1002/2015GL063083.

Krasting, J. P., A. J. Broccoli, K. W. Dixon, and J. R. Lanzante, 2013: Future changes in Northern Hemisphere snowfall. J. Climate, 26, 7813-7828, https://doi.org/10.1175/JCLI-D-12-00832.1.

Lettenmaier, D. P., and T. Y. Gan, 1990: Hydrologic sensitivities of the Sacramento-San Joaquin River basin, California, to global warming. Water Resour. Res., 26, 69-86, https:// doi.org/10.1029/WR026i001p00069.

Mankin, J. S., and N. S. Diffenbaugh, 2015: Influence of temperature and precipitation variability on near-term snow trends. Climate Dyn., 45, 1099-1116, https://doi.org/10.1007/ s00382-014-2357-4.

Mock, C. J., 1996: Climatic controls and spatial variations of precipitation in the western United States. J. Climate, 9,
1111-1125, https://doi.org/10.1175/1520-0442(1996)009<1111: CCASVO $>2.0 . \mathrm{CO} ; 2$.

Mote, P. W., A. F. Hamlet, M. P. Clark, and D. P. Lettenmaier, 2005: Declining mountain snowpack in western North America. Bull. Amer. Meteor. Soc., 86, 39-49, https://doi.org/10.1175/ BAMS-86-1-39.

— , and Coauthors, 2016: Perspectives on the causes of exceptionally low 2015 snowpack in the western United States. Geophys. Res. Lett., 43, 10 980-10 988, https://doi.org/10.1002/ 2016 GL069965.

_ , S. Li, D. P. Lettenmaier, M. Xiao, and R. Engel, 2018: Dramatic declines in snowpack in the western US. Nat. Partner J. Climate Atmos. Sci., 1, 2, https://doi.org/10.1038/s41612-018-0012-1.

Pierce, D. W., and D. R. Cayan, 2013: The uneven response of different snow measures to human-induced climate warming. J. Climate, 26, 4148-4167, https://doi.org/10.1175/JCLI-D-12-00534.1.

Serreze, M. C., M. P. Clark, R. L. Armstrong, D. A. McGinnis, and R. S. Pulwarty, 1999: Characteristics of the western United States snowpack from snowpack telemetry (SNOTEL) data. Water Resour. Res., 35, 2145-2160, https://doi.org/10.1029/1999WR900090.

Skaugen, T., and I. H. Weltzien, 2016: A model for the spatial distribution of snow water equivalent parameterized from the spatial variability of precipitation. Cryosphere, 10, 1947-1963, https://doi.org/10.5194/tc-10-1947-2016.

Stewart, I. T., D. R. Cayan, and M. D. Dettinger, 2004: Changes in snowmelt runoff timing in western North America under a 'business as usual' climate change scenario. Climatic Change, 62, 217-232, https://doi.org/10.1023/B:CLIM.0000013702.22656.e8.

Westerling, A. L., H. G. Hidalgo, D. R. Cayan, and T. W. Swetnam, 2006: Warming and earlier spring increase western U.S. forest wildfire activity. Science, 313, 940-943, https://doi.org/10.1126/ science.1128834.

Wilks, D. S., 2011: Statistical Methods in the Atmospheric Sciences. 3rd ed. Elsevier, 676 pp. 